\title{
Dynamics of $f(R)$ gravity models and asymmetry of time
}

\author{
Murli Manohar Verma* and Bal Krishna Yadav \\ Department of Physics, University of Lucknow, Lucknow 226 007, India
}

(Dated: June 11, 2018)

\begin{abstract}
We solve the field equations of modified gravity for $f(R)$ model in metric formalism. Further, we obtain the fixed points of the dynamical system in phase space analysis of $f(R)$ models, both with and without the effects of radiation. Stability of these points is studied against perturbations in a smooth spatial background by applying the conditions on the eigenvalues of the matrix obtained in the linearized first-order differential equations. Following this, these fixed points are used for analysing the dynamics of the system during the radiation, matter and acceleration dominated phases of the universe. Certain linear and quadratic forms of $f(R)$ are determined from the geometrical and physical considerations and the behaviour of the scale factor is found for those forms. Further, we also determine the Hubble parameter $H(t)$, Ricci scalar $R$ for these cosmic phases. We show the emergence of an asymmetry of time from the dynamics of the scalar field exclusively owing to the $f(R)$ gravity in the Einstein frame that may lead to an arrow of time at a classical level.
\end{abstract}

PACS numbers: 98.80.-k, 95.36.+x, 04.50.-h

\section{INTRODUCTION}

The present state of the universe has been found to be in the phase of accelerated expansion[1]. There are several observational evidences on geometry and growth of structures such as the Supernovae Ia, Baryon Acoustic Osicllation (BAO), Cosmic Microwave Background anisotropies, weak gravitational lensing etc. [1] which indicate the presence of a hitherto unknown dark energy. By all reckoning, the explanation for present accelerated expansion of the universe is a major challenge in cosmology, even though there are many approaches to explain its dynamics. The simplest candidate for dark energy is the cosmological constant with a constant equation of state $(w=-1)[5$. However, there are two main difficulties associated with the cosmological constant - (i) the fine tuning problem and (ii) the coincidence problem. Besides, there exist two basic approaches attmpting to explain dark energy. The first approach is based on modified matter models. In this approach $T_{\mu \nu}$ in the Einstein equations must include an exotic matter component like quintessence, k-essence, Phantom energy etc. 6 6] that takes the form of dark energy and so the resposibility of causing acceleration. The second approach is through the so-called modified gravity models wherein the latetime accelerated cosmic expansion is realized without requiring the explicit dark energy component in the universe. In these models, we have a wide range of $f(R)$ gravity [10, scalar-tensor theories, Gauss-Bonnet models, braneworld models etc. 11-13. Specifcally, in this paper we present an analysis of $f(R)$ models, where one modifies the laws of gravity by replacing the scalar curvature $R$ of the Hilbert's action, or $R-2 \Lambda$, as one includes in the standard $\Lambda$ CDM approach (with $\Lambda$ as the cosmological constant), by an arbitrary function of $R$ in the

\footnotetext{
* sunilmmv@yahoo.com

$\dagger$ balkrishnalko@gmail.com
}

curvature part of the Lagrangian density. At present, there is no specific, known functional form of $R$ which may satisfy all the observational conditions of cosmological viability ranging from the radiation dominated matter to the ongoing accelerated phase. Therefore, we study the stability conditions for the respective eras and determine the corresponding forms of $f(R)$. By solving the field equations for different forms of $f(R)$, the scale factor of expansion is thus determined. From here we find the scalar curvature $R$ and compare them in different eras. This can lead to the determination of a time-ordering of various epochs, dominated by radiation, matter and dark energy (as a modification of gravity), respectively, throughout the evolution of the universe.

In section II, the fixed points of the dynamical system are determined within the framework of $f(R)$ models in metric formalism. To study the exclusive effects of radiation, in sections III and IV the properties and stability of the fixed points of the dynamical system are found without and with radiation. Section $\mathrm{V}$ comprises of analysis of the behaviour of $f(R)$, scale factor $a(t)$, Hubble parameter $H(t)$ (under various conditions) and scalar curvature $R$ in radiation dominated phase. In sections VI and VII we attempt to determine the form of $f(R)$, scale factor $a(t)$ and scalar curvature $R$ for matter dominated and the present accelerated expansion dominated phases, respectively. Together, the time ordering may be used further to determine an arrow of time through the cosmic evolution in section VIII. Finally, we conclude our results in section IX.

\section{FIELD EQUATIONS AND PHASE SPACE DYNAMICS}

In $f(R)$ gravity we obtain the field equations in metric formalism, where the variation of the action is taken with respect to $g_{\mu \nu}$ related to the connections $\Gamma_{\beta \gamma}^{\alpha}$ in the usual sense (unlike the Palatini formalism where they are 
treated as mutually independent). We consider the field equations in the background of spatially flat FriedmannLemaitre-Robertson-Walker (FLRW) spacetime with a metric

$$
d s^{2}=-d t^{2}+a^{2}(t)\left[d r^{2}+r^{2}\left(d \theta^{2}+\sin ^{2} \theta d \phi^{2}\right)\right]
$$

where $a(t)$ is time dependent scale factor and the speed of light $c=1$. Correspondingly, the Ricci scalar $R$ is given by

$$
R=6\left(2 H^{2}+\dot{H}\right)
$$

where $H(=\dot{a} / a)$ is the Hubble parameter and an overdot represents the derivative with respect to time. The total action using an arbitrary function $f(R)$ in the Jordan frame is given by

$$
\mathcal{A}=\frac{1}{2 \kappa^{2}} \int \sqrt{-g} f(R) d^{4} x+\mathcal{A}_{m}
$$

where $\mathcal{A}_{m}$ is the action for relativistic and non-relativistic matter, $\kappa^{2}=8 \pi G$ and $g$ is the determinant of the metric tensor $g_{\mu \nu}$. Varying the action (3) with respect to $g_{\mu \nu}$, the field equations obtained are

$$
\begin{array}{r}
F(R) R_{\mu \nu}-\frac{1}{2} f(R) g_{\mu \nu} \\
-\nabla_{\mu} \nabla \nu F(R)+g_{\mu \nu} \square F(R)=\kappa^{2} T_{\mu \nu},
\end{array}
$$

where $F(R) \equiv \frac{\partial f}{\partial R}$ and $T_{\mu \nu}$ is the energy-momentum tensor for matter. From the above equation (4) and its trace we arrive at the the following ones

$$
\begin{gathered}
3 F H^{2}=\kappa^{2}\left(\rho_{m}+\rho_{r}\right)+\frac{(F R-f)}{2}-3 H \dot{F} \\
-2 F \dot{H}=\kappa^{2}\left(\rho_{m}+\frac{4}{3} \rho_{r}\right)+\ddot{F}-H \dot{F}
\end{gathered}
$$

where $\rho_{m}$ and $\rho_{r}$ are the energy densities of matter and radiation, respectively.

With four (dimensionless) variables defined as

$$
\begin{gathered}
x_{1} \equiv-\frac{\dot{F}}{F H} \\
x_{2} \equiv-\frac{f}{6 F H^{2}} \\
x_{3} \equiv \frac{R}{6 H^{2}} \\
x_{4} \equiv \frac{\kappa^{2} \rho_{r}}{3 F H^{2}}
\end{gathered}
$$

the effective equation of state for this system is defined by

$$
w_{e f f}=-1-\frac{2}{3} \frac{\dot{H}}{H^{2}}=-\frac{1}{3}\left(2 x_{3}-1\right)
$$

Differentiation of these variables $(7, \sqrt{10})$ with respect to $N=\ln a(t)$ gives

$$
\begin{gathered}
\frac{d x_{1}}{d N}=-1-x_{3}-3 x_{2}+x_{1}^{2}-x_{1} x_{3}+x_{4} \\
\frac{d x_{2}}{d N}=\frac{x_{1} x_{3}}{m}-x_{2}\left(2 x_{3}-4-x_{1}\right) \\
\frac{d x_{3}}{d N}=-\frac{x_{1} x_{3}}{m}-2 x_{3}\left(x_{3}-2\right) \\
\frac{d x_{4}}{d N}=-2 x_{3} x_{4}+x_{1} x_{4}
\end{gathered}
$$

where

$$
\begin{gathered}
m \equiv \frac{d \log F}{d \log R}=\frac{R f_{, R R}}{f_{, R}}, \\
q \equiv-\frac{d \log f}{d \log R}=-\frac{R f_{, R}}{f}=\frac{x_{3}}{x_{2}}
\end{gathered}
$$

where $f_{, R} \equiv \frac{d f}{d R}$ and $f_{, R R} \equiv \frac{d^{2} f}{d R^{2}}$. The fixed points of the system are obtained by equating the equations $[12-15$ to zero. Thus, the points are given by

$$
\begin{aligned}
& P_{1}:\left(x_{1}, x_{2}, x_{3}, x_{4}\right)=(0,-1,2,0) \text {, } \\
& \Omega_{m}=0, w_{e f f}=-1 \\
& P_{2}:\left(x_{1}, x_{2}, x_{3}, x_{4}\right)=(-1,0,0,0) \text {, } \\
& \Omega_{m}=2, w_{e f f}=\frac{1}{3} \\
& P_{3}:\left(x_{1}, x_{2}, x_{3}, x_{4}\right)=(1,0,0,0) \text {, } \\
& \Omega_{m}=0, w_{e f f}=\frac{1}{3} \\
& P_{4}:\left(x_{1}, x_{2}, x_{3}, x_{4}\right)=(-4,5,0,0) \text {, } \\
& \Omega_{m}=0, w_{e f f}=\frac{1}{3}, \\
& P_{5}:\left(x_{1}, x_{2}, x_{3}, x_{4}\right)= \\
& \left(\frac{3 m}{1+m},-\frac{1+4 m}{2(1+m)^{2}}, \frac{1+4 m}{2(1+m)}, 0\right) \text {, } \\
& \Omega_{m}=1-\frac{m(7+10 m)}{2(1+m)^{2}} \text {, } \\
& w_{e f f}=-\frac{m}{(1+m)} \text {, } \\
& P_{6}:\left(x_{1}, x_{2}, x_{3}, x_{4}\right)= \\
& \left(\frac{2(1-m)}{1+2 m}, \frac{1-4 m}{m(1+2 m)},-\frac{(1-4 m)(1+m)}{m(1+2 m)}, 0\right) \text {, } \\
& \Omega_{m}=0, \\
& w_{e f f}=\frac{2-5 m-6 m^{2}}{3 m(1+2 m)}
\end{aligned}
$$




$$
\begin{array}{r}
P_{7}:\left(x_{1}, x_{2}, x_{3}, x_{4}\right)=(0,0,0,1) \\
\Omega_{m}=0, \quad w_{\text {eff }}=\frac{1}{3} \\
P_{8}:\left(x_{1}, x_{2}, x_{3}, x_{4}\right)= \\
\left(\frac{4 m}{1+m},-\frac{2 m}{(1+m)^{2}}, \frac{2 m}{1+m}, \frac{1-2 m-5 m^{2}}{(1+m)^{2}}\right), \\
\Omega_{m}=0, \quad w_{e f f}=\frac{1-3 m}{3+3 m}
\end{array}
$$

\section{FIXED POINTS WITHOUT RADIATION}

First, we consider the properties and stability of these fixed points in the absence of radiation. For stability about the fixed points $\left(x_{1}, x_{2}, x_{3}\right)$ we invoke time dependent linear perturbations $\delta x_{i}(i=1,2,3)$ around the points in a smooth spatial background. Linearization of the equations 12 14 gives first order differential equations

$$
\frac{d}{d N}\left(\begin{array}{l}
\delta x_{1} \\
\delta x_{2} \\
\delta x_{3}
\end{array}\right)=M\left(\begin{array}{l}
\delta x_{1} \\
\delta x_{2} \\
\delta x_{3}
\end{array}\right),
$$

where $M$ is a $3 \times 3$ matrix whose components depend upon $x_{1}, x_{2}$ and $x_{3}$. Stability of each fixed point depends upon the eigenvalues of the matrix $M$ obtained by taking linear perturbations around that specific point. In the absence of radiation, we have only six fixed points $P_{1}-P_{6}$ as below.

(1) Point $P_{1}:(0,-1,2)$ corresponds to de-Sitter point. Here $w_{\text {eff }}=-1$ and eigenvalues corresponding to this point are

$$
-3,-\frac{3}{2} \pm \frac{\sqrt{25-\frac{16}{m}}}{2}
$$

$P_{1}$ is stable when real parts of all the eigenvalues is negative. Hence condition for stability is $0<$ $m(q=-2)<1$, otherwise it is a saddle point. So this point can be taken as an acceleration point.

(2) Point $P_{2}:(-1,0,0)$ is denoted by $\phi$-matterdominated ( $\phi \mathrm{MDE}$ ) epoch. The eigenvalues of the $3 \times 3$ matrix of perturbations about $P_{2}$ are given by

$$
\sqrt{\left(7+\frac{1}{m}-\frac{m^{\prime}}{m^{2}} q(1+q)\right)^{2}-4\left(12+\frac{1}{m}-\frac{m^{\prime}}{m^{2}} q(1+q)\right.} \mp
$$

where $m^{\prime}$ is derivative of $m$ w.r.t. $q$. If $m$ is constant, then eigenvalues are $-2,3,4+\frac{1}{m}$. In this case $P_{2}$ is a saddle point because eigenvalues are negative and positive.

$P_{2}$ can not be a matter dominated point because $\Omega_{m}=2$ and $w_{\text {eff }}=\frac{1}{3}$.

(3) Point $P_{3}:(1,0,0)$ is the kinetic point. The eigenvalues corresponding to this point are

$$
\sqrt{\left(9-\frac{1}{2}\left[9+\frac{1}{m}-\frac{m^{\prime}}{m^{2}} q(1+q) \mp\right.\right.}
$$

If $m$ is constant, the eigenvalues are 2,5,4- $\frac{1}{m}$. In this case $P_{3}$ is unstable for $m<0$ and $m>\frac{1}{4}$ and a saddle otherwise.

(4) Point $P_{4}:(-4,5,0)$ has eigenvalues:

$$
-5,-3,4\left(1+\frac{1}{m}\right)
$$

It is stable for $-1<m<0$ and saddle otherwise. This point cannot be used as a radiation or a matter dominated point.

(5) Point $P_{5}:\left(\frac{3 m}{1+m},-\frac{1+4 m}{2(1+m)^{2}}, \frac{1+4 m}{2(1+m)}\right)$ can be regarded as a standard matter point in the limit $m \rightarrow 0$. In this limit $\Omega_{m}=1$ and $a \propto t^{\frac{2}{3}}$. Hence necessary condition for this point to be a standard matter point is

$$
m(q=-1)=0 .
$$

Eigenvalues corresponding to point $P_{5}$ are given by

$$
\frac{3\left(1+m^{\prime}\right),}{-3 m \pm \sqrt{m\left(256 m^{3}+160 m^{2}-31 m-16\right)}}
$$

For a cosmologically viable trajectory, we want a saddle matter point. Hence, the condition for a saddle matter epoch is given by

$$
\begin{array}{r}
m(q \leq-1)>0, m^{\prime}(q \leq-1)>-1, \\
m(q=-1)=0
\end{array}
$$

(6) Point $P_{6}:\left(\frac{2(1-m)}{1+2 m}, \frac{1-4 m}{m(1+2 m)},-\frac{(1-4 m)(1+m)}{m(1+2 m)}\right)$ can also be an acceleration dominated point. The eigenvalues corresponding to this point are:

$-4+\frac{1}{m}, \frac{2-3 m-8 m^{2}}{m(1+2 m)},-\frac{2\left(m^{2}-1\right)\left(1+m^{\prime}\right)}{m(1+2 m)}$

Stability of this point depends on both $m$ and $m^{\prime}$. The condition of acceleration $\left(w_{\text {eff }}<-\frac{1}{3}\right)$ depends on the value of $m$. 


\section{FIXED POINTS WITH RADIATION}

Next, we include the radiation with other components of universe as a realistic case for our further study. In this case we have eight fixed points. Stability about the fixed points $\left(x_{1}, x_{2}, x_{3}, x_{4}\right)$ is determined in the same way as in absence of radiation. Here, we have $4 \times 4$ matrix of linear perturbations about each fixed point and four eigenvalues.

(1) Point $P_{1}$ corresponds to de-Sitter point. Here $w_{\text {eff }}=-1$ and eigenvalues corresponding to this point are

$$
-4,-3,-\frac{3}{2} \pm \frac{\sqrt{25-\frac{16}{m}}}{2}
$$

In the presence of radiation, we have an eigenvalue -4 in addition to those in the absence of radiation. Since this eigenvalue is negative, therefore the condition of stability is the same in both cases. $P_{1}$ is stable when $0<m(q=-2)<1$. This point may be taken as an acceleration point. The condition of stability for this point is same as in the case of without radiation because here we have only an extra eigenvalue -4 , which is negative.

(2) Point $P_{2}$ is denoted by $\phi$-matter-dominated ( $\phi$ MDE) epoch. The eigenvalues corresponding to this point are given by

$$
\sqrt{-2,-1, \frac{1}{2}\left[7+\frac{1}{m}-\frac{m^{\prime}}{m^{2}} q(1+q) \mp\right.}
$$

$P_{2}$ is either saddle or stable point. In this case $P_{2}$ can not be a matter point because $\Omega_{m}=2$ and $w_{\text {eff }}=\frac{1}{3}$.

(3) Point $P_{3}$ is known as kinetic point. The eigenvalues for the $4 \times 4$ matrix of perturbations about this point are

$$
\begin{array}{r}
1,2, \frac{1}{2}\left[9+\frac{1}{m}-\frac{m^{\prime}}{m^{2}} q(1+q) \mp\right. \\
\sqrt{\left.\left(9-\frac{1}{m}+\frac{m^{\prime}}{m^{2}} q(1+q)\right)^{2}-4\left(20-\frac{5}{m}-\frac{m^{\prime}}{m^{2}} q(5+4 q)\right)\right]}
\end{array}
$$

If $m$ is constant, the eigenvalues corresponding to this point are $2,5,4-\frac{1}{m}$. In this case $P_{3}$ is unstable for $m<0$ and $m>\frac{1}{4}$ and a saddle otherwise.
(4) Point $P_{4}$ has eigenvalues

$$
-5,-4,-3,4\left(1+\frac{1}{m}\right)
$$

It is stable for $-1<m<0$ and saddle otherwise. This point can not be use as a radiation or a matter dominated point.

(5) Point $P_{5}$ can be regarded as a standard matter point in the limit $m \rightarrow 0$. Eigenvalues for point $P_{5}$ are given by

$$
\frac{-3 m \pm \sqrt{m\left(256 m^{3}+160 m^{2}-31 m-16\right)}}{4 m(m+1)}
$$

where $m^{\prime}$ is derivative of $m$ w.r.t. q. For a cosmologically viable trajectory, we want a saddle matter point. The condition for a saddle matter epoch is given by

$$
\begin{array}{r}
m(q \leq-1)>0, m^{\prime}(q \leq-1)>-1 \\
m(q=-1)=0
\end{array}
$$

(6) Point $P_{6}$ can also be an acceleration dominated point. The eigenvalues corresponding to this point are given by

$$
\begin{array}{r}
-\frac{2\left(-1+2 m+5 m^{2}\right)}{m(1+2 m)},-4+\frac{1}{m}, \\
\frac{2-3 m-8 m^{2}}{m(1+2 m)},-\frac{2\left(m^{2}-1\right)\left(1+m^{\prime}\right)}{m(1+2 m)}
\end{array}
$$

Stability of this point depends on both $m$ and $m^{\prime}$. Condition of acceleration $\left(w_{e f f}<-\frac{1}{3}\right)$ depends on the value of $m$.

(7) Point $P_{7}$ corresponds to a standard radiation point. The eigenvalues of $P_{7}$ for constant $m$ are $4,4,1,-1$. Thus, $P_{7}$ is a saddle point.

(8) Point $P_{8}$ also is a radiation point. In this case dark energy is non-zero, therefore $P_{8}$ is acceptable as a radiation point. The eigenvalues of $P_{8}$ are given by

$$
1,4\left(1+m^{\prime}\right), \frac{m-1 \pm \sqrt{81 m^{2}+30 m-15}}{2(m+1)}
$$

Point $P_{8}$ is a saddle point in the limit $m \rightarrow 0$. The acceptable radiation dominated point $P_{8}$ lies at point $(0,-1)$ in the $(m, q)$ plane. 


\section{DYNAMICS OF RADIATION DOMINATED PHASE}

For radiation dominated era, phase space analysis shows that we can find a radiation point in the limit $m \rightarrow 0$ at point $P_{8}$. This point lies on the line $m=-q-1$ in the $(m, q)$ plane. Hence, the necessary condition for this point to exist as an exact standard radiation point is given by

$$
m(q=-1) \approx 0 .
$$

From definition of $q$ and the above condition, the form of $f(R)$ for radiation dominated era is given by

$$
f(R)=\alpha R
$$

where $\alpha$ is an integration constant. The standard radiation point is obtained by substitution of $m \approx 0$ in the radiation point of $m(q)$ curve. In this condition, the effective equation of state is

$$
w_{e f f}=\frac{1}{3}
$$

Using equations (11) and 45, the Hubble parameter is given by

$$
H(t)=\frac{1}{\left(2 t-c_{1}\right)}
$$

where $c_{1}$ is an integration constant.

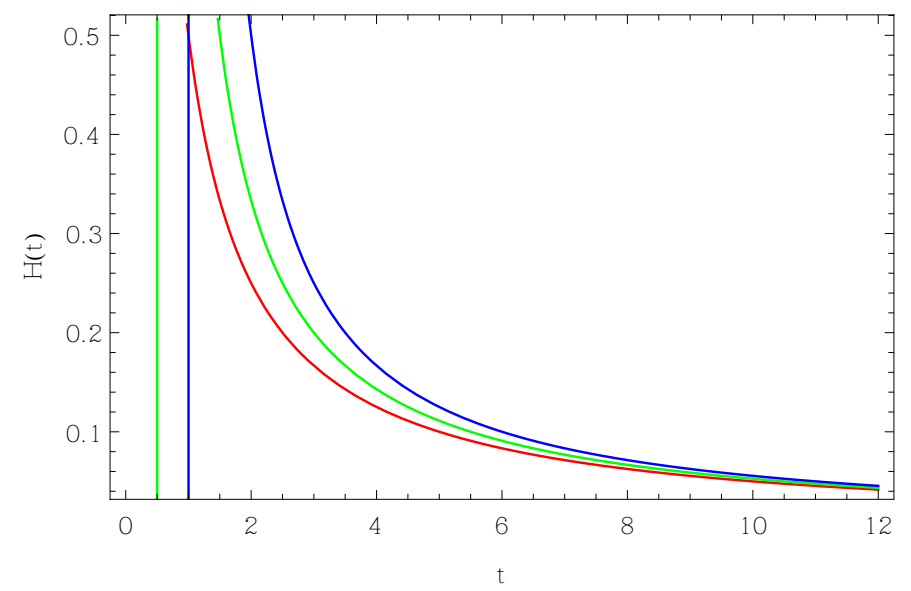

FIG. 1. Plot for variation of the Hubble parameter $H(t)$ with cosmic time $t$ in radiation dominated phase. The red, green and blue curves correspond to $c_{1}=0, c_{1}=1, c_{1}=2$, respectively.

The scale factor for this era is given by

$$
a(t)=c_{2}\left(2 t-c_{1}\right)^{\frac{1}{2}}
$$

where $c_{2}$ is another integration constant.

In radiation dominated phase we confirm that the scale factor $a(t) \propto t^{\frac{1}{2}}$, which is same as in the case of standard

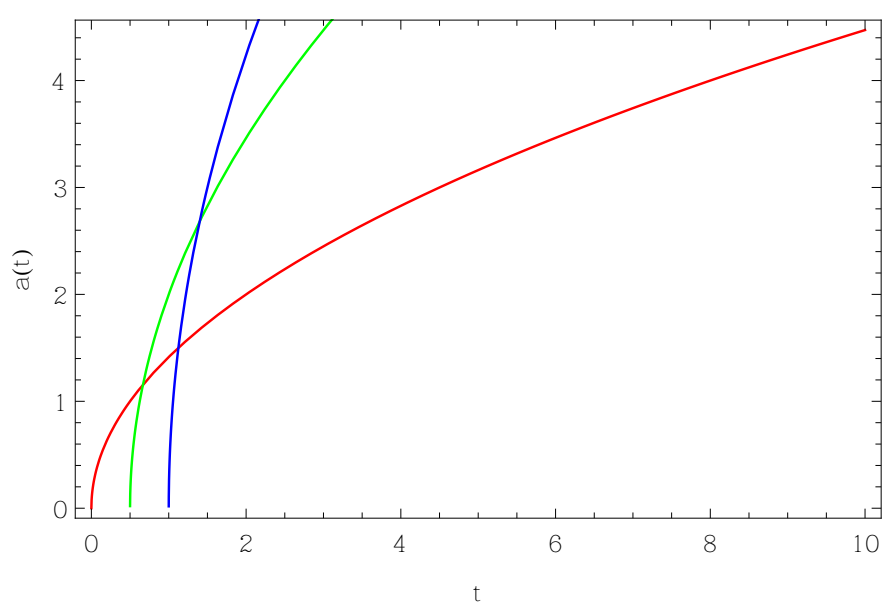

FIG. 2. Plot for variation of the scale factor $a(t)$ with cosmic time $(0 \leq t \leq 10)$ in radiation dominated phase. The red, green and blue curves correspond to $\left(c_{1}, c_{2}\right) \equiv(0,1) ;\left(c_{1}, c_{2}\right) \equiv$ $(1,2) ;\left(c_{1}, c_{2}\right) \equiv(2,3)$, respectively.

model. Figures (1) and (2) show variation the Hubble parameter $H(t)$ and scale factor $a(t)$ with time $t$ in radiation phase. As expected, the Ricci scalar $R$ for radiation dominated era is given by

$$
R=0
$$

\section{DYNAMICS OF MATTER DOMINATED ERA}

From the field equations (5) and (6) we obtain the following equation

$$
-\frac{\kappa^{2} \rho_{r}}{3}+3 F H^{2}+F \dot{H}-\frac{f}{2}-2 H \dot{F}-\ddot{F}=0
$$

In phase space analysis of dynamical system, there is a point $P_{5}$ which represents a standard matter era in the limit $m \rightarrow 0$. In matter dominated phase of the Universe

$$
m(q=-1) \approx 0
$$

Using the definition of $q$ or $m$, the form of $f(R)$ is given by

$$
f(R)=\beta R
$$

where $\beta$ is an integration constant. Thus, in matter dominated phase the form of $f(R)$ is similar as in the case of radiation dominated phase.

In matter dominated phase, we neglect the energy density of radiation i.e. $\rho_{r}=0$. For $f(R)=\beta R, F=\beta$ and therefore $\dot{F}=0$. Using equations 49 and 2 and these values of $F$ and $\dot{F}$, the time evolution of the Hubble parameter is expressed as

$$
H(t)=\frac{1}{\left(\frac{3}{2} t-c_{3}\right)}
$$




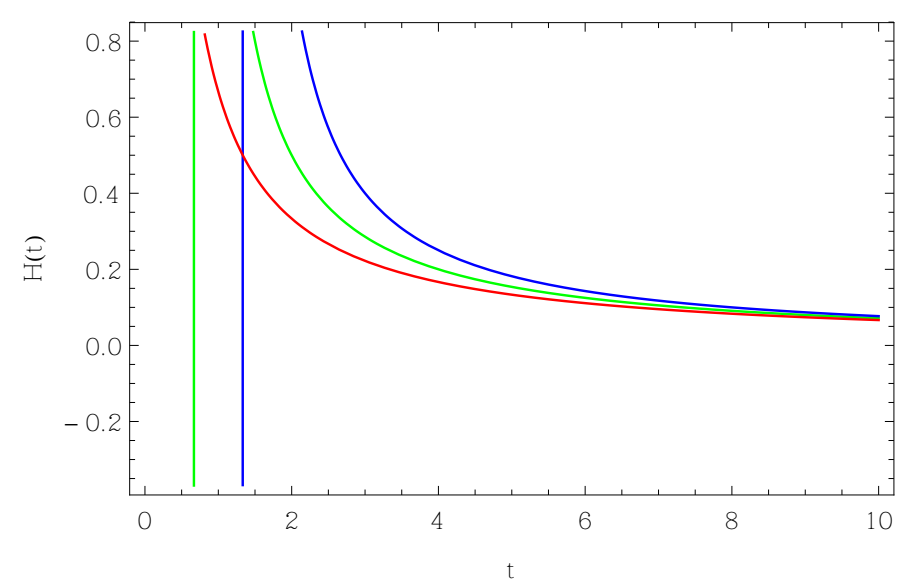

FIG. 3. Plot for variation of the Hubble parameter $H(t)$ with cosmic time $t$ in matter dominated phase.The red, green and blue curves correspond to $c_{3}=0, c_{3}=1, c_{3}=2$, respectively.

where $c_{3}$ is an integration constant.

The scale factor in this phase is given by the expression

$$
a(t)=c_{4}\left(\frac{3}{2} t-c_{3}\right)^{\frac{2}{3}}
$$

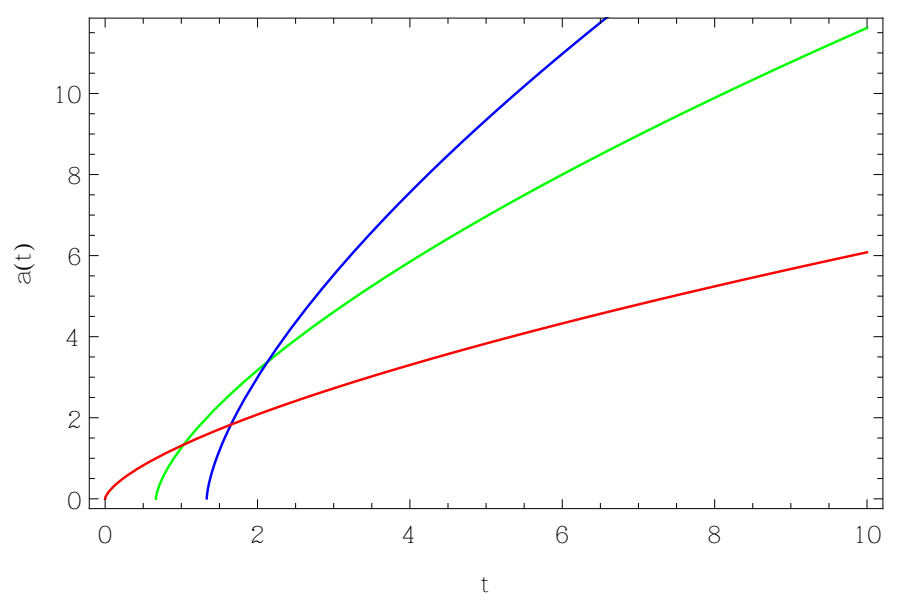

FIG. 4. Plot for variation of the scale factor $a(t)$ with cosmic time $t$ in matter dominated phase.The red, green and blue curves correspond to $\left(c_{3}, c_{4}\right) \equiv(0,1) ;\left(c_{3}, c_{4}\right) \equiv$ $(1,2) ;\left(c_{3}, c_{4}\right) \equiv(2,3)$, respectively.

From equations (2) and 52 the Ricci scalar in matter dominated phase is given by

$$
R=\frac{3}{\left(\frac{3}{2} t-c_{3}\right)^{2}}
$$

The variation of Hubble parameter $H(t)$, scale factor $a(t)$ and Ricci scalar $R$, with time is plotted in figures (3), (4), and (5) respectively. Hubble parameter $H(t)$, scale factor $a(t)$, and Ricci scalar $R$ in this phase can

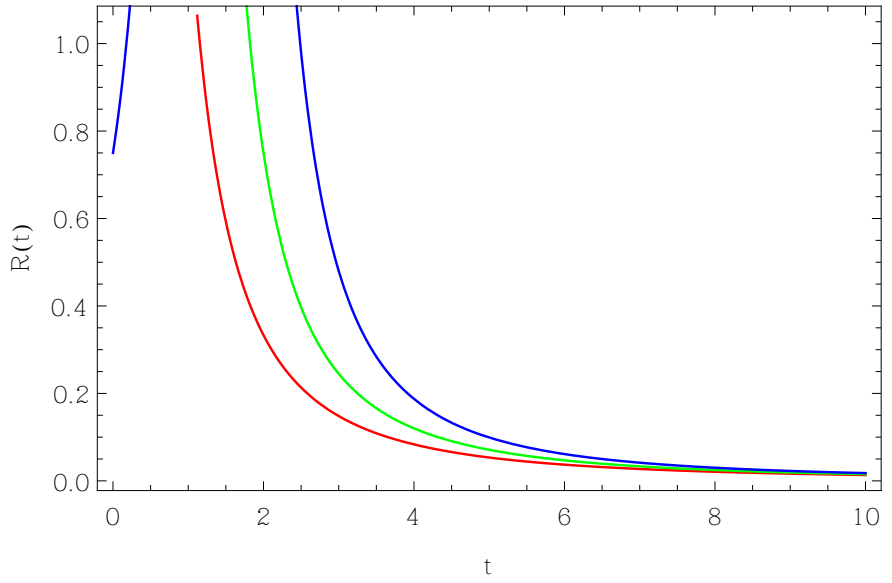

FIG. 5. Plot for variation of Ricci scalar $R$ with cosmic time $t$ in matter dominated phase. The red, green and blue curves correspond to $\left(c_{3}, c_{4}\right) \equiv(0,1) ;\left(c_{3}, c_{4}\right) \equiv(1,2) ;\left(c_{3}, c_{4}\right) \equiv$ $(2,3)$, respectively.

also be calculated by the same procedure as we followed in the radiation era. Expressions for these parameters are same in both approaches. For $m \approx 0$, the effective equation of state is given by

$$
w_{\text {eff }}=0
$$

These expressions of scale factor $a(t)$, Hubble parameter $H(t)$, and Ricci scalar $R$ in matter dominated phase are similar to the expressions of standard $(\Lambda \mathrm{CDM})$ model.

\section{DYNAMICS OF ACCELERATED EXPANSION DOMINATED PHASE}

In the phase space analysis, there is a point $P_{1}$, for which effective equation of state is

$$
w_{\text {eff }}=-1
$$

This point is called de Sitter point. If we take de Sitter expansion, this point is stable when $0<m<1$ at $q=-2$. Now from the definition of $q$, the form of $f(R)$ in this phase is given by

$$
f(R)=\gamma R^{2}
$$

We have the effective equation of state

$$
w_{\text {eff }}=-1-\frac{2}{3} \frac{\dot{H}}{H^{2}}
$$

Now, using equations (56) and (58), in this phase we get the constant value of the Hubble parameter as

$$
H(t)=c_{5}
$$

where $c_{5}$ is an integration constant. Therefore, the Ricci scalar in this phase is given by

$$
R=12 c_{5}^{2}
$$


Using the expression of Hubble parameter $H(t)$, the scale factor is given by

$$
a(t)=e^{c_{5} t+c_{6}}
$$

where $c_{6}$ is another integration constant. We can also find out these parameters using equations $(49)$ and (2) in the spatially flat universe.

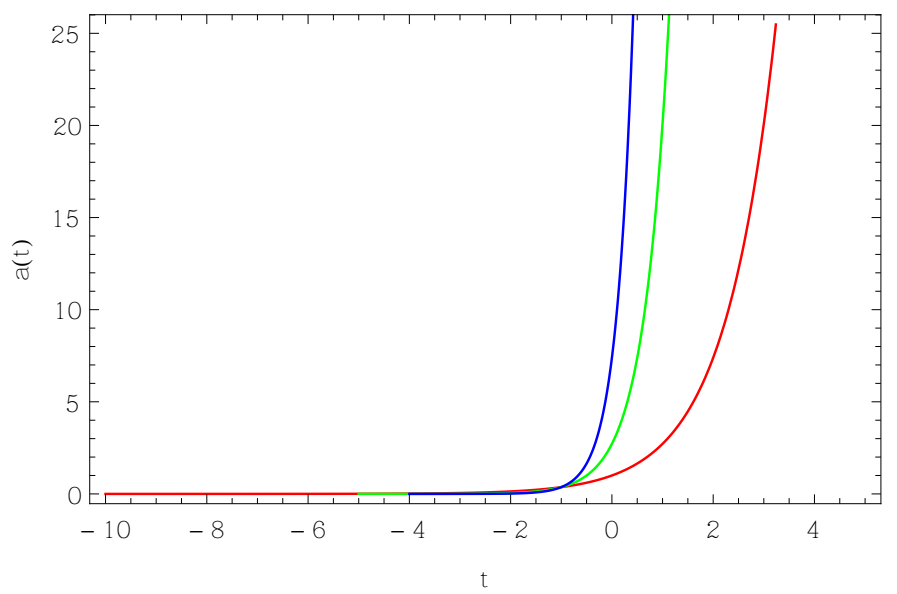

FIG. 6. Plot for variation of the scale factor $a(t)$ with cosmic time $t$ in acceleration dominated phase. The red, green and blue curves correspond to $\left(c_{5}, c_{6}\right) \equiv(1,0) ;\left(c_{5}, c_{6}\right) \equiv$ $(2,1) ;\left(c_{5}, c_{6}\right) \equiv(3,2)$, respectively.

Here, figure (6) shows the variation of scale factor $a(t)$ with time. It is clear that expansion in this phase is exponential. This behaviour is found to be similar to the case of the standard $(\Lambda \mathrm{CDM})$ model.

\section{ASYMMETRY OF TIME}

We rewrite the action (3) in the form

$$
\mathcal{A}=\int \sqrt{-g}\left(\frac{1}{2 \kappa^{2}} F R-U\right) d^{4} x+\mathcal{A}_{m},
$$

where

$$
U=\frac{F R-f}{2 \kappa^{2}} .
$$

It is possible to derive an action in the Einstein frame under the conformal transformation

$$
\tilde{g}_{\mu \nu}=\Omega^{2} g_{\mu \nu},
$$

where $\Omega^{2}$ is the conformal factor and a tilde denotes the quantities pertaining to the Einstein frame. The corresponding Ricci scalars in the two frames are related as

$$
R=\Omega^{2}\left(\tilde{R}+6 \square \omega-6 \tilde{g}^{\mu \nu} \partial_{\mu} \omega \partial_{\nu} \omega\right),
$$

where

$$
\omega \equiv \ln \Omega, \partial_{\mu} \omega \equiv \frac{\partial \omega}{\partial \tilde{x}^{\mu}}, \square \omega \equiv \frac{1}{\sqrt{-\tilde{g}}} \partial_{\mu}\left(\sqrt{-\tilde{g}} \tilde{g}^{\mu \nu} \partial_{\nu} \omega \nmid 66\right)
$$

Thus, the action 62 is transformed as

$$
\mathcal{A}=\int d^{4} x \sqrt{-\tilde{g}}\left[\frac{1}{2 \kappa^{2}} F \Omega^{-2}\left(\tilde{R}+6 \square \omega-6 \tilde{g}^{\mu \nu} \partial_{\mu} \omega \partial_{\nu} \omega\right)-\Omega^{-4} U\right]
$$

The linear action in $\tilde{R}$ can be written by choosing

$$
\Omega^{2}=F .
$$

We consider a new scalar field $\phi$ defined by

$$
\kappa \phi \equiv \sqrt{\frac{3}{2}} \ln F .
$$

Using these relations the action in Einstein frame is found as [15]

$$
\mathcal{A}=\int d^{4} x \sqrt{-\tilde{g}}\left[\frac{1}{2 \kappa^{2}} \tilde{R}-\frac{1}{2} \tilde{g}^{\mu \nu} \partial_{\mu} \phi \partial_{\nu} \phi-V(\phi)\right]+\mathcal{A}_{m} .
$$

where

$$
V(\phi)=\frac{U}{F^{2}}=\frac{F R-f}{2 \kappa^{2} F^{2}} .
$$

On varying the action 70 w.r.t. $\phi$ in the absence of matter (relativistic and non-relativistic, both), we get

$$
\frac{d^{2} \phi}{d \tilde{t}^{2}}+3 \tilde{H} \frac{d \phi}{d \tilde{t}}+V_{, \phi}=0 .
$$

with $V_{, \phi}$ implying the usual derivative w.r.t. $\phi$. The energy density and pressure of the above homogeneous scalar field, respectively, are

$$
\rho=\frac{1}{2} \dot{\phi}^{2}+V(\phi) ; p=\frac{1}{2} \dot{\phi}^{2}-V(\phi),
$$

while the scalar field equation of motion is given by 72 .

Tolman described a cyclic universe with progressively larger cycles, assuming the presence of a viscous fluid with pressure

$$
p=p_{0}-3 \zeta H,
$$

where $p_{0}$ is the equilibrium pressure and $\zeta$ is the coefficient of bulk viscosity [14. It is clear from equation (74) that $p<p_{0}$ during expansion $(H>0)$ whereas $p>p_{0}$ during contraction. This asymmetry during the expanding and contracting phases results in the growth of both energy and entropy. This increase in entropy makes the amplitude of successive expansion cycles larger leading to a arrow of time.

In our discussion of $f(R)$ gravity models, the term $3 \tilde{H} \frac{d \phi}{d \tilde{t}}$ in 72 behaves like friction and damps the motion of the scalar field when the universe $(H>0)$. In 
a contracting universe, however, this term behaves like anti-friction and accelerates the motion of the scalar field. A scalar field with the potential $V=M^{2} \phi^{2}$ gives $p \simeq-\rho$ when $H>0$ and $p \simeq \rho$ when $H<0$. These results are in conformity with those of Tolman.

Further, we derive different potentials in all the phases of the Universe. In radiation dominated phase we have $f(R)=\alpha R$, therefore, the potential given by equation 71 is $V(\phi)=0$ for this phase. Similarly, for matter dominated phase we have $f(R)=\beta R$ and $V(\phi)=0$. For accelerated expansion phase, the form of the Lagrangian is given by including $f(R)=\gamma R^{2}$ and the potential for this phase is $V(\phi)=\frac{1}{8 \gamma \kappa^{2}}$.

The scalar field given by equation 69 gives $\kappa \phi=$ $\sqrt{\frac{3}{2}} \ln \alpha$ for matter dominated phase and $\kappa \phi=\sqrt{\frac{3}{2}} \ln \beta$ for radiation dominated phase. For accelerated expansion phase

$$
\kappa \phi=\sqrt{\frac{3}{2}} \ln (2 \gamma R)
$$

The general solution of the equation 72 for the potential $V(\phi)=\frac{1}{2} M^{2} \phi^{2}$ is given by

$$
\begin{array}{r}
\phi=\frac{\phi_{0}}{2} \exp (-\Psi t)\left(1+\frac{\Psi}{\sqrt{\Psi^{2}-M^{2}}}\right) \exp \left(\sqrt{\Psi^{2}-M^{2}}\right) t+ \\
\frac{\phi_{0}}{2} \exp (-\Psi t)\left(1-\frac{\Psi}{\sqrt{\Psi^{2}-M^{2}}}\right) \exp \left(-\sqrt{\Psi^{2}-M^{2}}\right) t
\end{array}
$$

where $\phi_{0}$ is the maximum value of scalar field $\phi$ at $t=0$ ( $t$ being the time in the Einstein frame henceforth), $\Psi=$ $\frac{3 \tilde{H}}{2}$, and $M$ is the mass of the scalar field. In this solution three cases arises depending on the value of $\sqrt{\Psi^{2}-M^{2}}$ as discussed below.

\section{A. Case (i): $M^{2}>\Psi^{2}$}

In this case the solution is given by

$$
\phi=\frac{\phi_{0} M}{\omega} \exp (-\Psi t) \sin (\omega t+\theta)
$$

where $\omega=\sqrt{M^{2}-\Psi^{2}}$ and $\theta$ is the phase angle. If we take $\Psi$ as a positive constant, then figure (7) shows the nature of scalar field in this case. These oscillations are damped harmonic oscillations. In the perturbations due to local gravity, a very large field mass corresponds to smaller deviations from the standard $\Lambda$ CDM model.

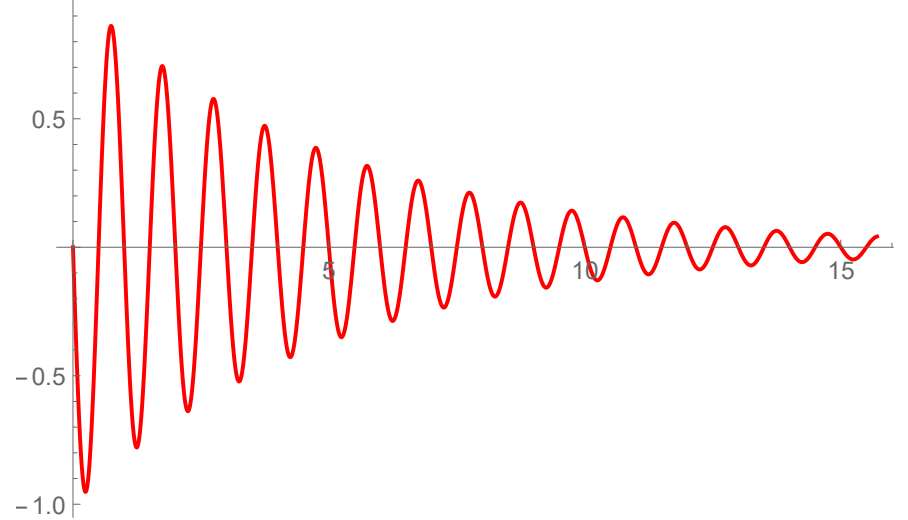

FIG. 7. Plot for variation of scalar field $\phi$ along $Y$-axis with time $t$ along $X$-axis in case $M^{2}>\Psi^{2}$. Here, we have taken $\Psi=\frac{1}{5}, \frac{\phi_{0} M}{\omega}=1, \omega=2 \pi$ and $\theta=\pi$.

\section{B. Case (ii): $M^{2}<\Psi^{2}$}

The general solution of field equation is given by

$$
\begin{array}{r}
\phi=\frac{\phi_{0}}{2} \exp (-\Psi t)\left(1+\frac{\Psi}{\sqrt{\Psi^{2}-M^{2}}}\right) \exp \left(\sqrt{\Psi^{2}-M^{2}}\right) t+ \\
\frac{\phi_{0}}{2} \exp (-\Psi t)\left(1-\frac{\Psi}{\sqrt{\Psi^{2}-M^{2}}}\right) \exp \left(-\sqrt{\Psi^{2}-M^{2}}\right) t
\end{array}
$$

In this case $\left(\Psi^{2}-M^{2}\right)$ is a positive quantity and there is an exponential term with negative power. So, the field dies off exponentially with time. There is no oscillation and the motion become over-damped. Figure (8) shows the behaviour of the scalar field $\phi$ with time $t$.

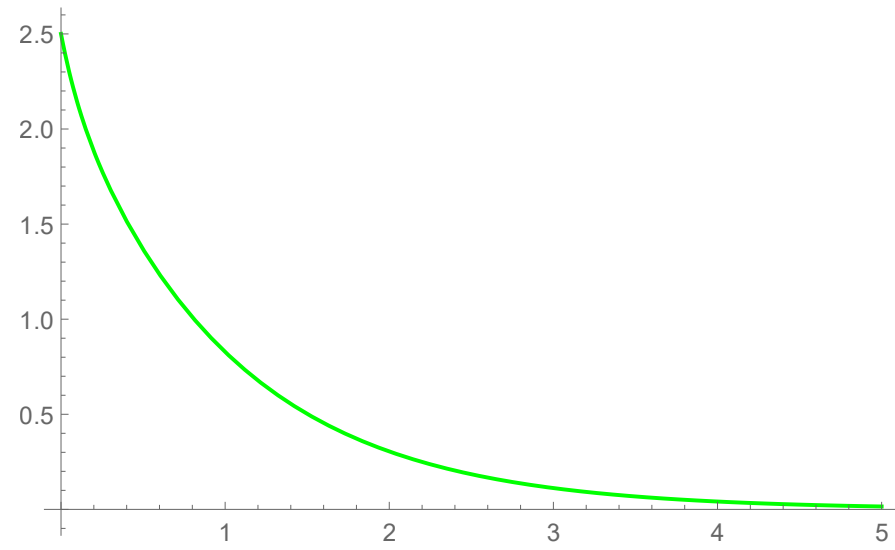

FIG. 8. Plot for variation of the scalar field $\phi$ along $Y$-axis with time $t$ along $X$-axis in case $M^{2}<\Psi^{2}$. The values of the parameters are as $\Psi=5, M=3$, and $\phi_{0}=2$. 


$$
\text { C. Case (iii): } M^{2}=\Psi^{2}
$$

This is a special case, appearing as the critical damping of the scalar field. The solution is given as

$$
\phi=\phi_{0} \exp (-\Psi t)(1+\Psi t)
$$

indicating an aperiodic damping. Figure (9) shows the nature of the scalar field $\phi$ with the positive $\Psi$.

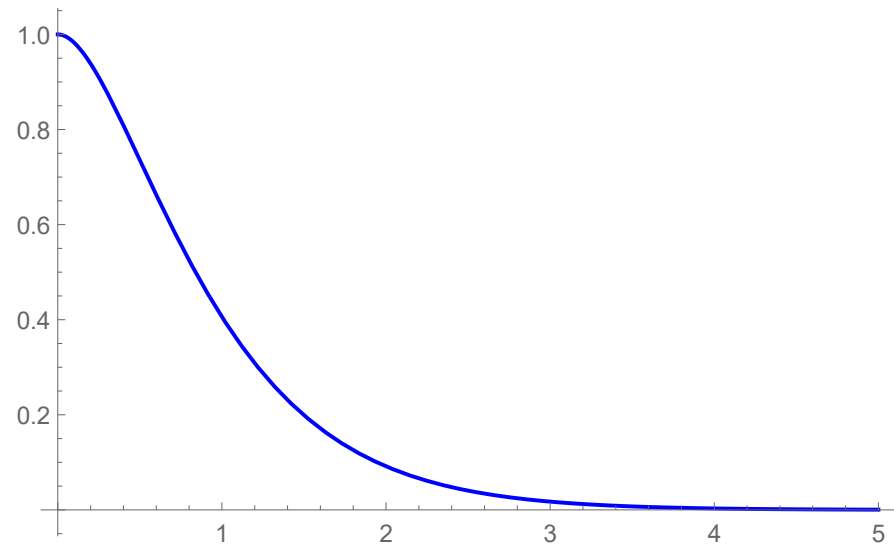

FIG. 9. Plot for variation of scalar field $\phi$ along $Y$-axis with time $t$ along $X$-axis in case $M^{2}=\Psi^{2}$. Here, we have taken $\Psi=2$ and $\phi_{0}=1$.

It is clear that in all the above cases we get a damping either periodic or aperiodic that owes its relation to $\Psi=\frac{3 \tilde{H}}{2}$ and mass $M$ of the scalar field $\phi$. When $\tilde{H}$ is positive and constant, $\Psi$ damps the scalar field. When $\Psi$ is negative and constant, the motion of the scalar field accelerates in all the three cases.

We also find that the scalar field is not symmetric under the reversal of time i.e. $(t \leftrightarrow-t)$ for positive and negative $\Psi=\frac{3 \tilde{H}}{2}$. This dissipation of the scalar field indicates a crucial asymmetry in time in form of its arrow from past to future.

\section{CONCLUSION}

While describing the $f(R)$ models of modified gravity, we have studied the properties and stability of the fixed points of the dynamical system against the time dependent perturbations in a smooth spatial background. We discussed the role of radiation in our analysis and compared it with the case without radiation. It is found that the nature of the fixed points with radiation remains unaltered as that without radiation (except that with radiation we have the emergence of an extra eigenvalue for each point). (Of course, the future discussion would bring out an analysis of the fixed points against spatial perturbations as well). We have determined the forms of $f(R)$ for different phases of the universe, over radiation, matter and acceleration dominated eras, by using the necessary conditions for the phase space analysis to reach eventually at a cosmologically viable model. The scale factor $a(t)$, the Hubble parameter $H(t)$, Ricci scalar $R$ have been determined for these phases, with a view that their ordering over the entire evolution of the universe may explain the emergence of an arrow of time, more comprehensively in a future study. While these model parameters are found to be consistent with $\Lambda$ CDM model, the crucial issue is that the scalar field $\phi$, that owes its origin exclusively to $f(R)$ gravity, may be invoked to explain the arrow of time based on its explicit asymmetry on a classical level. While the scalar-tensor theories may have the forms of potentials matching with $f(R)$, the results obtained on the stability conditions and their ordering extending through the overall history may not be reproduced in such theories. We would further explore this fundamental aspect of the nature of time and its observational viability within the modified gravity sector.

\section{ACKNOWLEDGMENTS}

MMV thanks Edward Kolb at the Kavli Institute of Cosmological Physics, the University of Chicago, USA for hosting the visit, and Varun Sahni at the Inter-University Centre for Astronomy and Astrophysics (IUCAA), Pune, India for discussion and facilities. Both authors thank IUCAA for the support under the associateship programme.
[1] A. G. Riess et al., (Supernova Search Team Collaboration), Astron. J. 116, 1009 (1998).

[2] B. Schmidt et al., Astrophys. J. 507, 46 (1998).

[3] S. Perlmutter et al., Astrophys. J. 517, 565 (1999).

[4] D. N. Spergel et al., Astrophys. J. Suppl. 148, 97 (2003).

[5] T. Padmanabhan, Phys. Rept., textbf380, 235 (2003).
[6] R. R. Caldwell, R. Dave, and P. J. Steinhardt, Phys. Rev. Lett. 80, 1582 (1998).

[7] S. Capozziello, Int. J. Mod. Phys. D 11, 483 (2002)

[8] T. Chiba, T. Okabe, and M. Yamaguchi, Phys. Rev. D 62, 023511 (2000).

[9] R. R. Caldwell, Phys. Lett. B 545, 23 (2002).

[10] A. A. Starobinski, Phys. Lett. B 91, 99(1980). 
[11] M. Kunz and D. Sapone, Phys. Rev. Lett. 98, 121301 (2007).

[12] L. Amendola, R. Gannouji, D. Polarski and S. Tsujikawa, Phys. Rev. D 75, 083504 (2007).
[13] V. Sahni and Y. Shantov, JCAP 0311, 014 (2003).

[14] R. C. Tolman, Relativity, Thermodynamics and Cosmology (Oxford: Clarendon) (1934).

[15] L. Amendola and S. Tsujikawa, Dark energy: Theory and Observations (Cambridge) (2010). 